\title{
A Case of Anti-Glomerular Basement Membrane Antibody Disease in Siblings
}

\author{
Pen Li ${ }^{\mathrm{a}}$, Pierre-Marc Villeneuve ${ }^{\mathrm{a}, \mathrm{b}, \mathrm{c}}$
}

\begin{abstract}
Anti-glomerular basement membrane (anti-GBM) antibody disease, also known as Goodpasture syndrome, is associated with the presence of antibodies against type 4 collagen. Increasing evidence supports the role of human leukocyte antigen (HLA) genes in the pathogenesis of this disease. A 48-year-old Caucasian male was admitted to the intensive care unit with diffuse alveolar hemorrhage and rapidly progressive glomerulonephritis. Serology demonstrated high anti-GBM antibodies (291.8 U/L). Renal biopsy showed crescentic glomerulonephritis involving 16 of 19 glomeruli with strong linear and diffuse IgG staining confirming the diagnosis of anti-GBM antibody disease. The patient's sister presented with pulmonary-renal syndrome at age 29 due to the same illness. Subsequent HLA typing revealed that our patient was homozygous for DRB1*15:01, an allele strongly associated with anti-GBM antibody disease. To our knowledge, this is the first case report of anti-GBM antibody disease in a patient who is homozygous for DRB $1 * 15: 01$ with a sibling who had the same diagnosis. Our case highlights the importance of HLA genes in the pathogenesis of this disease.
\end{abstract}

Keywords: Anti-GBM disease; Goodpasture disease; Familial; Genetic; Siblings; Relative

\section{Introduction}

Anti-glomerular basement membrane (anti-GBM) antibody disease, also known as Goodpasture syndrome, is an extremely rare disease with an estimated incidence of 0.1 per million [1]. It involves the production of short-lived circulating antibodies against the GBM [2]. Specifically, anti-GBM antibodies target the $\mathrm{NC} 1$ domain of the $\alpha-3$ chain of type 4

Manuscript accepted for publication February 17, 2015

aDepartment of Medicine, University of Alberta, Edmonton, Canada bivision of Critical Care Medicine, University of Alberta, Edmonton, Canada ${ }^{\mathrm{c} C}$ Corresponding Author: Pierre-Marc Villeneuve, Department of Medicine, University of Alberta, Room 2-124 Clinical Sciences Building, 8440-112 Street, Edmonton, AB T6G 2B7, Canada. Email: villeneu@ualberta.ca

doi: http://dx.doi.org/10.14740/wjnu198w collagen $(\alpha 3(\mathrm{IV}) \mathrm{NC} 1)$ [3], although other $\alpha$-chains have been described as targets [4]. $\alpha 3$ (IV)NC1 is highly expressed in the alveoli and GBM; therefore, patients classically present with a pulmonary-renal syndrome characterized by diffuse alveolar hemorrhage and a rapidly progressive glomerulonephritis. Increasing evidence supports the role of genetic factors in the pathogenesis of anti-GBM antibody disease. Human leukocyte antigen (HLA) genes and in particular the DRB1*15:01 allele is frequently and most strongly associated with this disease [5-8]. Other positive HLA associations have been found with DRB1*04 and DRB1*03. Conversely, negative associations have been found with DRB1*07 and DRB1*01 [8].

This case report describes classic anti-GBM antibody disease in a patient homozygous for DRB1*15:01, who had a sister with the same disease. To our knowledge, there are currently no reported cases of anti-GBM antibody disease in a patient with a first degree relative with the same disease or any reported case of anti-GBM disease in multiple family members.

\section{Case Report}

A previously healthy 48 -year-old Canadian Caucasian male was transferred from a community hospital with hemoptysis and hypoxemic respiratory failure. He presented with a 2-week history of an influenza-like illness associated with myalgias, fevers, sore throat, and sinusitis. During the preceeding week, he had gross hematuria, hemoptysis, and progressive shortness of breath.

At presentation to our tertiary care hospital, his oxygen saturation was $95 \%$ on $15 \mathrm{~L}$ of oxygen delivered by non-rebreather mask. He was hypertensive with a blood pressure of $150 / 90$ and was tachycardic. His work of breathing was mildly increased with a respiratory rate of 22; auscultation revealed normal breath sounds. Cardiac and abdominal exams were unremarkable, and there was no rash. His frontal and maxillary sinuses were tender to palpation. He deteriorated to require $100 \% \mathrm{FiO}_{2}$ delivered via high flow nasal cannula and was subsequently admitted to the intensive care unit.

Initial laboratory testing demonstrated anemia, acute kidney injury with hematuria (Table 1). Chest X-ray and computed tomography (CT) of the chest revealed bilateral ground glass opacities consistent with diffuse alveolar hemorrhage 
Table 1. Laboratory Investigations

\begin{tabular}{ll}
\hline Lab test & Result \\
\hline Hemoglobin & $72 \mathrm{~g} / \mathrm{L}$ \\
White blood cell count & $10.4\left(10^{9} / \mathrm{L}\right)$ \\
Platelets & $188\left(10^{9} / \mathrm{L}\right)$ \\
Erythrocyte sedimentation rate & $107 \mathrm{~mm} / \mathrm{h}$ \\
Ferritin & $1,053 \mu \mathrm{g} / \mathrm{L}$ \\
Lactate dehydrogenase & $571 \mathrm{U} / \mathrm{L}$ \\
Albumin & $23 \mathrm{~g} / \mathrm{L}$ \\
Creatinine & $465 \mu \mathrm{mol} / \mathrm{L}$ \\
Urinalysis & $3+$ hemoglobin, 3+ protein \\
Protein to creatinine ratio & $236.44 \mathrm{mg} / \mathrm{mmol}$ \\
Immunology & Positive: \\
& Anti-GBM Ab 291.8 U/L \\
& Negative: \\
& Anti-nuclear Ab \\
& Anti-dsDNA \\
& Rheumatoid factor \\
& Anti-cyclic citrullinated \\
peptide Ab \\
Anti-neutrophil cytoplasmic \\
Ab \\
ENA screen \\
Anti-streptolysis O Ab \\
\\
\end{tabular}

(Fig. 1). CT scan of the sinuses only found minimal thickening of his maxillary, frontal, ethmoid, and sphenoid sinuses.

Further laboratory testing revealed a high titre of antiGBM antibodies (291.8 U/L) and absent anti-neutrophil cytoplasmic antibodies. An urgent kidney biopsy was performed and showed a necrotizing and crescentic glomerulonephritis involving 16 of 19 glomeruli. Immunofluorescence had strong linear and diffuse IgG staining consistent with anti-GBM antibody disease.

Detailedhistory revealed that the patient's older sister developed anti-GBM antibody disease in 1981 at the age of 29. Records show she also presented with a pulmonary-renal syndrome and required intubation in the ICU. While she initially required hemodialysis, there was ultimately renal recovery.
The patient's sister remained in remission until she passed away at age 48 of unrelated cardiac disease. Given the family history, we performed HLA typing. Our patient was homozygous for DRB1*15:01, an allele strongly associated with anti-GBM antibody disease. We were unable to perform HLA typing of his sister as she was deceased.

Our patient was initially treated with a pulse dose of methylprednisolone $500 \mathrm{mg}$ for 3 days followed by prednisone 1 $\mathrm{mg} / \mathrm{kg}$. On the second day of admission, plasmapheresis was initiated for a total course of seven treatments and cyclophosphamide was started at $150 \mathrm{mg}$ orally daily. As a result, his anti-GBM antibody waned. Subsequent chest X-rays demonstrated resolving pulmonary infiltrates with concurrent decrease in oxygenation requirements. The patient did not need intubation. Unfortunately, he required intermittent hemodialysis for his progressive renal failure. He was discharged after a 2 weeks hospital stay. At 3-month follow-up the patient remains dialysis dependent.

\section{Discussion}

The rarity of anti-GBM antibody disease makes its manifestation in two siblings particularly notable. No other instances of familial clustering of this disease have been reported in the literature. In the case of our patient, he is homozygous for DRB1*15:01, an HLA closely associated with anti-GBM antibody disease [5-8]. The HLA class II molecules encoded by $\mathrm{DRB} 1 * 15: 01$ have a high affinity for $\alpha 3(\mathrm{IV}) \mathrm{NC} 1$ peptides [8]. By being homozygous for DRB1*15:01, the patient may be especially vulnerable to developing antibodies against $\alpha 3$ (IV) $\mathrm{NC} 1$. Although we could not perform HLA typing of his sister, she must carry at least one copy of the DRB1*15:01 allele and possibly could be homozygous.

DRB1*15:01 is present in up to $10-15 \%$ of Caucasian patients [8], yet anti-GBM antibody disease is far less frequent. Clearly, other genetic and environmental factors are important in the development of this disease. An inciting stimulus, such as exposure to hydrocarbons [9], tobacco smoke [10], and pulmonary infections [2] is thought to be required to reveal the cryptic epitopes to the immune system which leads to antibody production. It has been suggested that urinary tract infections, lithotripsy, or pre-existing glomerulonephritis may also do this

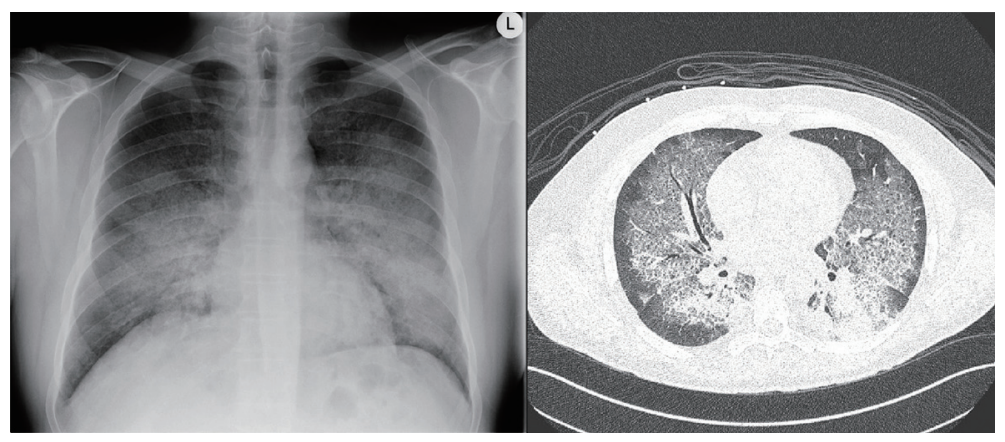

Figure 1. Chest X-ray and computed tomography of the chest demonstrating ground glass opacities consistent with diffuse alveolar hemorrhage in anti-glomerular basement membrane antibody disease. 
[2]. However the link between these common conditions and the rare occurrence of anti-GBM antibody disease is still unproven. The trigger in our patient may have been a viral infection, although this is impossible to verify.

The DRB1*15:01 allele is associated with several other diseases. Our literature review yielded a case involving identical twins who were DRB1*15:01 homozygotes; one developed anti-GBM antibody disease and the other had systemic lupus erythematosus [11]. Other diseases associated with this allele include multiple sclerosis [12], allergic bronchopulmonary aspergillosis [13], and cervical cancer [14].

In summary, we present the first clinical report of antiGBM antibody disease in a patient who is homozygous for DRB1*15:01, with a sibling who had the same disease. Our case highlights the importance of HLA type in the pathogenesis of anti-GBM antibody disease and possible genetic predisposition in some families.

\section{Acknowledgement}

None.

\section{Conflicts of Interest}

The authors declare that there is no conflict of interests regarding the publication of this paper.

\section{References}

1. Bolton WK. Goodpasture's syndrome. Kidney Int. 1996;50(5):1753-1766.

2. Pusey CD. Anti-glomerular basement membrane disease. Kidney Int. 2003;64(4):1535-1550.

3. Kalluri R, Wilson CB, Weber M, Gunwar S, Chonko AM, Neilson EG, Hudson BG. Identification of the alpha 3 chain of type IV collagen as the common autoantigen in antibasement membrane disease and Goodpasture syndrome. J Am Soc Nephrol. 1995;6(4):11781185.

4. Zhao J, Cui Z, Yang R, Jia XY, Zhang Y, Zhao MH. Anti- glomerular basement membrane autoantibodies against different target antigens are associated with disease severity. Kidney Int. 2009;76(10):1108-1115.

5. Fisher M, Pusey CD, Vaughan RW, Rees AJ. Susceptibility to anti-glomerular basement membrane disease is strongly associated with HLA-DRB1 genes. Kidney Int. 1997;51(1):222-229.

6. Kitagawa W, Imai H, Komatsuda A, Maki N, Wakui H, Hiki Y, Sugiyama S. The HLA-DRB1*1501 allele is prevalent among Japanese patients with anti-glomerular basement membrane antibody-mediated disease. Nephrol Dial Transplant. 2008;23(10):3126-3129.

7. Yang R, Cui Z, Zhao J, Zhao MH. The role of HLADRB1 alleles on susceptibility of Chinese patients with anti-GBM disease. Clin Immunol. 2009;133(2):245-250.

8. Phelps RG, Rees AJ. The HLA complex in Goodpasture's disease: a model for analyzing susceptibility to autoimmunity. Kidney Int. 1999;56(5):1638-1653.

9. Stevenson A, Yaqoob M, Mason H, Pai P, Bell GM. Biochemical markers of basement membrane disturbances and occupational exposure to hydrocarbons and mixed solvents. QJM. 1995;88(1):23-28.

10. Donaghy M, Rees AJ. Cigarette smoking and lung haemorrhage in glomerulonephritis caused by autoantibodies to glomerular basement membrane. Lancet. 1983;2(8364):1390-1393.

11. Liu X, Wu Y, Yang Y, Wang J, Tao Y, Fu P, Hu Z. Identical twins:one with anti-glomerular basement membrane glomerulonephritis, the other with systemic lupus erythematosus. BMC Nephrol. 2013;14:277.

12. Quelvennec E, Bera O, Cabre P, Alizadeh M, Smadja D, Jugde F, Edan G, et al. Genetic and functional studies in multiple sclerosis patients from Martinique attest for a specific and direct role of the HLA-DR locus in the syndrome. Tissue Antigens. 2003;61(2):166-171.

13. Slavin RG, Hutcheson PS, Chauhan B, Bellone CJ. An overview of allergic bronchopulmonary aspergillosis with some new insights. Allergy Asthma Proc. 2004;25(6):395-399.

14. Apple RJ, Erlich HA, Klitz W, Manos MM, Becker TM, Wheeler CM. HLA DR-DQ associations with cervical carcinoma show papillomavirus-type specificity. Nat Genet. 1994;6(2):157-162. 